\title{
Evaluation of Residual Stress Determinations Conducted with Laser Ablation and Optical Displacement Measurement
}

\author{
Peter Weidmann ${ }^{1, a^{*}}$, Giancarlo Pedrini ${ }^{2, \text { b }}$, Venancio Martínez-García ${ }^{3, ~ c,}$ \\ Martin Wenzelburger ${ }^{3}$, Andreas Killinger ${ }^{3}$, Siegfried Schmauder ${ }^{1}$, Rainer Gadow ${ }^{3}$, \\ Wolfgang Osten ${ }^{2}$ \\ ${ }^{1}$ University of Stuttgart, IMWF, Pfaffenwaldring 32, 70569 Stuttgart, Germany \\ ${ }^{2}$ University of Stuttgart, ITO, Pfaffenwaldring 9, 70569 Stuttgart, Germany \\ ${ }^{3}$ University of Stuttgart, IFKB, Allmandring 7b, 70569 Stuttgart, Germany \\ apeter.weidmann@imwf.uni-stuttgart.de, bgiancarlo.pedrini@ito.uni-stuttgart.de, \\ cvenancio.martinez@gsame.uni-stuttgart.de
}

\section{Keywords: Residual Stress, Optical Determination, Laser Ablation}

\begin{abstract}
The presented work is related to the determination of residual stresses in ceramic coatings, where the material removal is done by laser ablation and the occurring surface deformation is measured with digital holography. Insights into the underlying assumptions and calculations to determine the depth dependent stress values from extensive displacement measurements shall be given. Moreover, the inhomogeneous elastic material properties and deviations from idealized removal geometries (in this case holes and notches) have to be considered. To cover all involved fields, i.e. thermodynamics and continuum mechanics, numerical studies for conventional and laser hole drillings were conducted and the ablation process was simulated. As the laser cannot produce ideal cylindrical or rectangular geometries, appropriate mathematical approximations are applied to choose a representative profile of the removed material. Furthermore, the calculation of stresses based on full field displacement measurements is done with an own program in several depth increments. These calculations are based on numerical calibration data for the elastically inhomogeneous compound of coating and substrate. Especially notch-like forms of material removal, where case sensitive weighting functions are applied for evaluation, can lead to reliable results. The physical experiments were done on aluminium plates with applied alumina layers.
\end{abstract}

\section{Introduction}

The reduction of manufacturing costs and enhancement of product lifetime are central goals for many modern research areas. Especially components which are supposed to withstand aggressive thermal, mechanical or chemical environments can be challenging in their production and maintenance. A reliable way to protect metals from high thermal and abrasive loads, i.e. turbine blades, can be achieved by coating them with suitable ceramics by atmospheric plasma spraying (APS). Hereby, a ceramic powder is injected into a plasma jet, molten and accelerated towards the specimen. During the impact of the liquid particle it is highly deformed and will quench almost immediately due to the comparably low heat capacity which leads to high tensile stresses in the solidified ceramic. When the coating is finished the whole component will bear a non-homogeneous temperature distribution, but even for a uniform temperature the differences in thermal expansion coefficients of substrate and coating will lead to further residual stresses during the cooling-down. While this residual stress cannot be avoided, it is possible to conduct the process in such ways, that a sometimes even beneficial state can be achieved, i.e. a compressive residual stress within the brittle coating. Nevertheless it is of great importance to know the existing stress state of the investigated part in order to ensure high quality products. A profound determination technique for this case is the incremental hole drilling (IHD). Hereby, a mechanical drill removes incrementally material out of the

(c) (1) Content from this work may be used under the terms of the Creative Commons Attribution 3.0 license. Any further distribution of this work must maintain attribution to the author(s) and the title of the work, journal citation and DOI. Published under license by Materials Research Forum LLC. 
stress afflicted component. As a new equilibrium is achieved, the area around the hole deforms. This deformation is mostly measured by a strain gauge and with a proper case sensitive calibration the underlying residual stress can be derived from it [1], [2].

The drawbacks of this method are the necessity of mechanical contact with the specimen, the specimen preparation with strain gauges and the overall time-consuming drilling operating. Therefore, a new approach shall be investigated, where the material removal is conducted via laser ablation and the occurring surface displacements will be measured by digital holography (DH).

\section{Boundary conditions and set-up}

The majority of investigations were conducted on an aluminium sample $(\mathrm{E}=68 \mathrm{GPa}, \mu=0.34)$ with a thickness of $6 \mathrm{~mm}$ on which a $200 \mu \mathrm{m} \mathrm{Al}_{2} \mathrm{O}_{3}$ coating $(\mathrm{E}=166 \mathrm{GPa}, \mu=0.23)$ was applied by APS. The specimens are initially grit blasted in order to enhance the adhesion between substrate and coating, which leads to a preliminary surface near hydrostatic residual compressive stress. Afterwards, the coating is applied and the overall residual stress distribution can be determined after the specimen has cooled down to room temperature (Fig. 1). For this determination a mechanical IHD operation was conducted, where an integral method (IM) evaluation scheme with a case sensitive calibration was applied [1]. Further numerical studies showed, that the high values after 200 $\mu \mathrm{m}$ depth might be mainly related to a plastification process due to the stress intensification around the hole. The relaxation of the coating tends to stress the aluminium $50 \%$ over its yield strength, which leads to an overestimation of the residual stress determination.

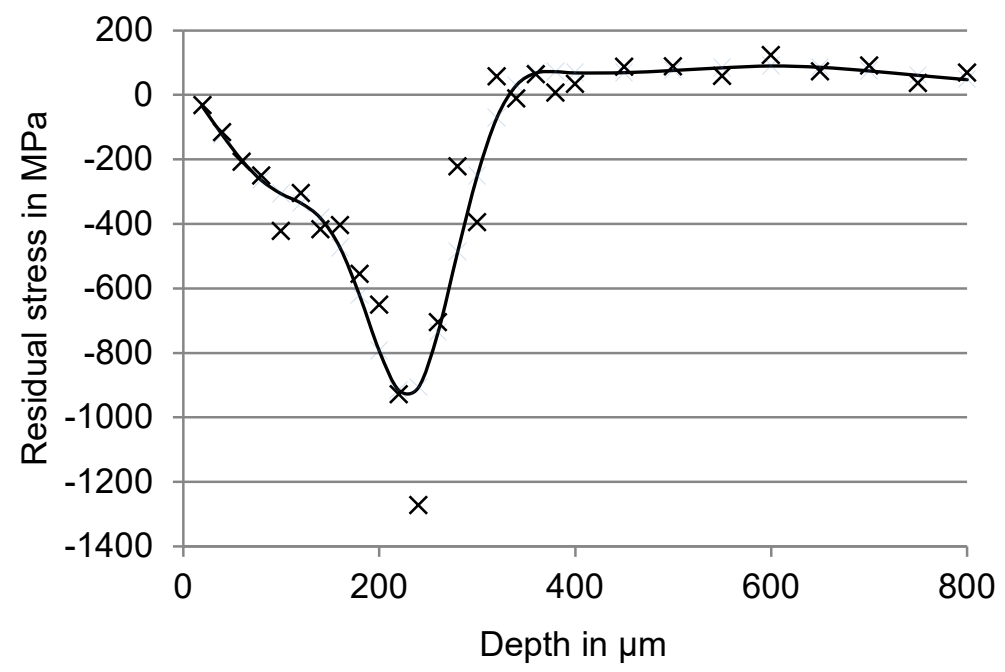

Fig. 1: Residual stress distribution, determined with classical IHD and case sensitive calibration

As the next step, the drilling and displacement measurement shall be done by optical manners. To measure the surface displacement, digital holography was applied. Hereby, green laser light (wavelength $532 \mathrm{~nm}$ ) is used to illuminate the specimen under at least one direction. The reflected light is overlaid with a reference beam, this leads to an interference pattern which is recorded with a CCD sensor. When the surface changes due to residual stress relaxation, the wavelength of reflected light also changes, which leads to another interference pattern. By overlaying reference- and finalpattern the underlying displacement can be calculated [3].

For the material ablation a SpitLight 600 laser from InnoLas $(\mathcal{C}$ with a wavelength of $1064 \mathrm{~nm}$, a pulse length of $7 \mathrm{~ns}$, a repetition rate of $20 \mathrm{~Hz}$ and an energy of $40 \mathrm{~mJ}$ was used. Due to the high energy densities $\left(>10^{6} \mathrm{~W} / \mathrm{cm}^{2}\right)$ the subjected material sublimates almost suddenly and a high back pressure builds up which accelerates the ablated material out of the hole. As this thermodynamic process imposes heat, a numerical simulation was conducted in order to investigate possible heating effects [4]. It was shown, that the majority of heat is transported out of the specimen by the 
sublimation of material. The resulting heat affected zone is smaller than $10 \mu \mathrm{m}$, which is also observed in corresponding micrographs of the holes cross-section (Fig. 2). Therefore, only a neglectable amount of heat is really imposed into the remaining material, which means that further surface strains are hereby not achieved.
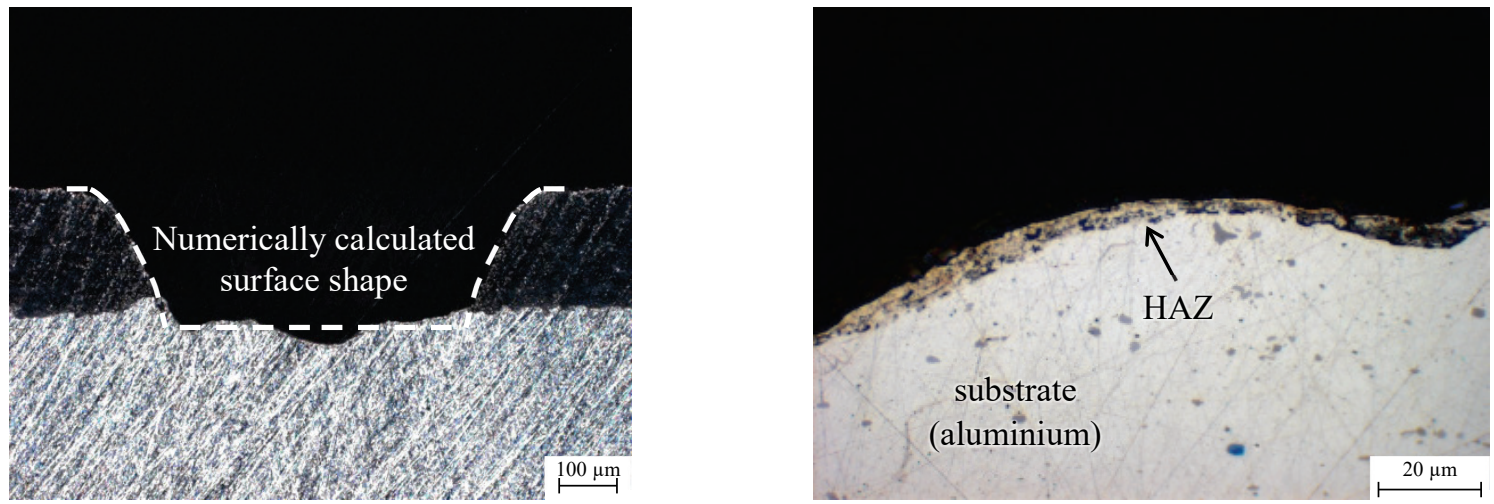

Fig. 2: Ablated hole shape for spot focused laser beam (left) and corresponding heat affected zone (HAZ) [5]

\section{Residual stress evaluation for hole-like structures}

The first residual stress determinations were conducted on simple hole-like structures like in Fig. 2. Here, an unshaped laser beam was used for the ablation process and led to a non-cylindrical structure. A well-known procedure to calculate the residual stress field of extensive displacement measurement was introduced by Schajer and Steinzig in 2005 [5], [6]. It relies on the integral method, where the radial profiles of pure shear and hydrostatic stress induced surface displacements are used for calibration. Hence, several finite-element-method (FEM) models have been made to calculate these profiles for the laser hole geometries in several depth steps. Therefore, white-light confocal microscopy images of the surface ( $x$ - $y$-plane) have been made, in order to evaluate the hole geometry for subsequent increments. Those images were then approximated with a best-fit-algorithm by frustums of a cone aka the equation

$$
\left(x-x_{m}\right)^{2}+\left(y-y_{m}\right)^{2}=[a / h(h-z)]^{2},
$$

where $x_{m}$ and $y_{m}$ are referring to the middle, $a$ to the radius and $h$ to the frustums height. Therefore, a convenient description of the material removal was achieved and the transfer into a FEM program could be eased. A more detailed description can be found in [7]. Despite the efforts, the resolution and deviation are not as good as the ones for classical IHD (Fig. 3). Yet, the qualitative trend is comparable to the IHD and, therefore, the feasibility of this method could be shown. But to enhance the method, especially the shape of the removed material must be improved.

\section{Residual stress evaluation for improved ablation of notch structures}

While the ablation of a simple hole structure doesn't need further equipment, it lacks necessary geometrical accuracy. Therefore, the system was enhanced with a spatial light modulator (SLM), in order to achieve material removal in more sophisticated forms. For hydrostatic residual stress states, like in most APS coatings, a stress determination in one direction is enough. Therefore, a notch was produced by laser ablation with considerable geometrical accuracy in several increments. One increment is achieved by applying approximately 200 pulses over the notch-length within one sequence. The displacement is then measured after every depth increment via digital holography perpendicular to the notch. Rigid body movements are simply filtered by adjusting the outer values regarding their angle and absolute displacements to zero. The basic evaluation scheme relies on the integral method [8]. That means a calibration for the given material and removal geometry has to be 
calculated step- and load-wise with proper FEM models in advance. For this reason several confocal microscopy images have been taken after different sequences to find the real notch depths. The achieved notch length was $1.1 \mathrm{~mm}$ for a width of $0.15 \mathrm{~mm}$, while the ablation depth per sequence was approximately $6.03 \mu \mathrm{m}$. The FEM calibration was done for increments with a thickness of 10 $\mu \mathrm{m}$.

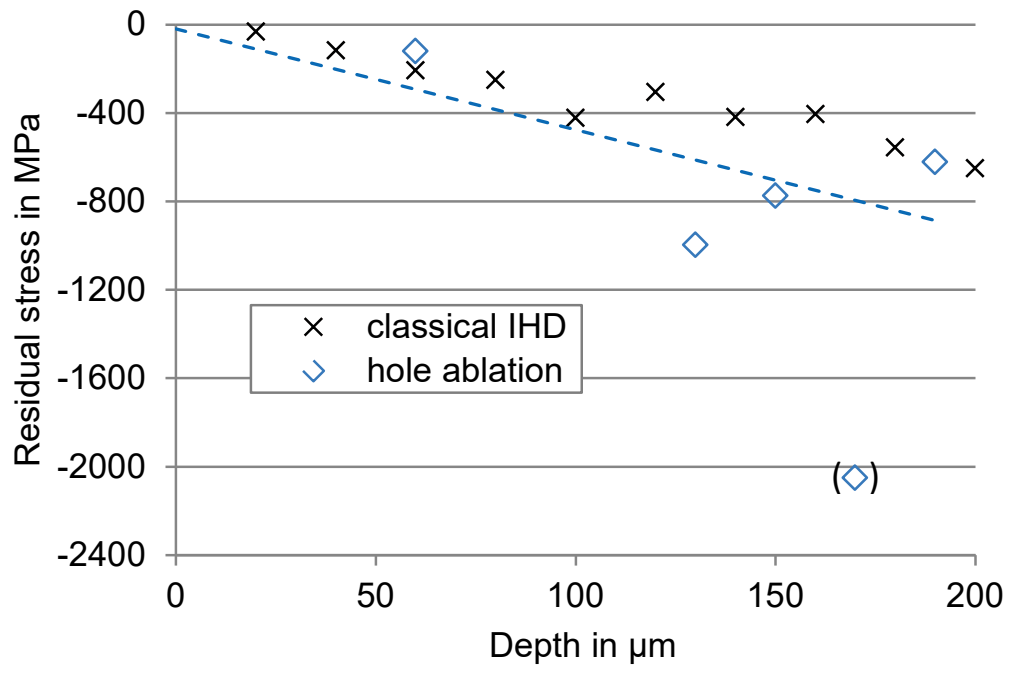

Fig. 3: Residual stress determination with incremental laser ablated hole-like structure

Compared to hole-like ablations, the relieved surface displacements are no longer radial symmetrical, why the formerly described evaluation cannot be applied. Of course, the values close to the notch tend to be the highest, while they gradually decrease towards the outside (Fig. 4). Hence, it is self-evident to weight the displacements according to their height, as for normal distributed absolute errors higher values are more reliable. To find a suitable weighting function the displacement field (along the $\mathrm{x}$ - and $\mathrm{y}$-direction) is represented by two functions: one along the parallel side of the notch as a power function

$$
f(y)=p_{1} \cdot y^{p_{2}}+p_{3}
$$

and one in the middle of the notch, parallel to the $\mathrm{x}$-axis as an arc tangent function

$$
f(x)=-p_{4} \cdot \operatorname{atan}\left(p_{5}\left(x-p_{6}\right)\right)+p_{7}
$$

The parameters $p_{1}$ to $p_{7}$ were identified with the commercial tool Matlab ${ }^{\odot}$. With this regression, a weighting distribution can simply be calculated by writing the values of $f(y)$ in columns of a matrix which has the size of the measurement field. The same is done for $f(x)$ in an equal matrix, but line wise. The product of both matrices leads to a measurement data based weighting distribution $F$, which should be normalized to its maximum value. Its shape is overall similar to the one of Fig. 4, but it is mirrored symmetrical to the x-axis. A weighted mean displacement for all measurement points $i$ can then easily be calculated by

$$
\overline{\mathrm{U}}=\sum_{\mathrm{i}}^{\mathrm{n}}\left[\mathrm{F}_{\mathrm{i}} \cdot \mathrm{U}_{\mathrm{i}}\right] / \sum_{\mathrm{i}}^{\mathrm{n}}\left[\mathrm{F}_{\mathrm{i}}\right]
$$

The same procedure has to be applied on the calibration fields $K$ in order to be able to calculate the residual stress distribution $\sigma$ with the matrix operation $\overline{\mathrm{K}} \cdot \sigma=\overline{\mathrm{U}}$. Hence, the displacement- and calibration-fields are described by their mean value over the same area. As it is difficult to ablate an 
exactly defined depth, it is reasonable to calculate the calibration coefficients $\overline{\mathrm{K}}$ for a given depth by a bivariate interpolation scheme [8]. After the primary residual stress distribution was determined, the results can be regularized with the Tikhonov regularization [9]. To investigate the effects of weighting, a numerical study for an incremental laser notch ablation was done. Therefore, a corresponding FEM model was used, where a residual stress of $200 \mathrm{MPa}$ was applied. The resulting surface displacements were then artificially spread by means of a Gaussian distribution with a standard deviation of $8 \mathrm{~nm}$. The resulting deviation of residual stress was $18 \mathrm{MPa}$. By using the proposed weighing, this deviation could be lowered to $12 \mathrm{MPa}$.

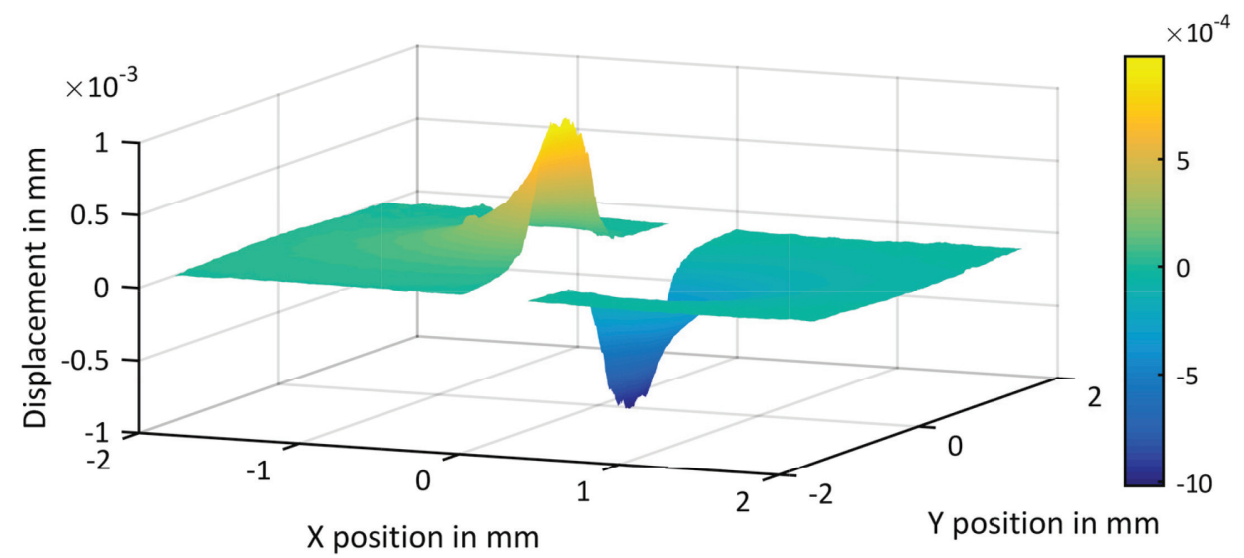

Fig. 4: Displacement field for an ablated notch at a depth of $0.12 \mathrm{~mm}$

In Fig. 5 the results of the residual stress determination with laser ablation material removal are plotted together with the results from the classical incremental hole drilling. The resolution is significantly better than for simple hole ablation (Fig. 3), while the deviation compared to the regularized data is acceptable.

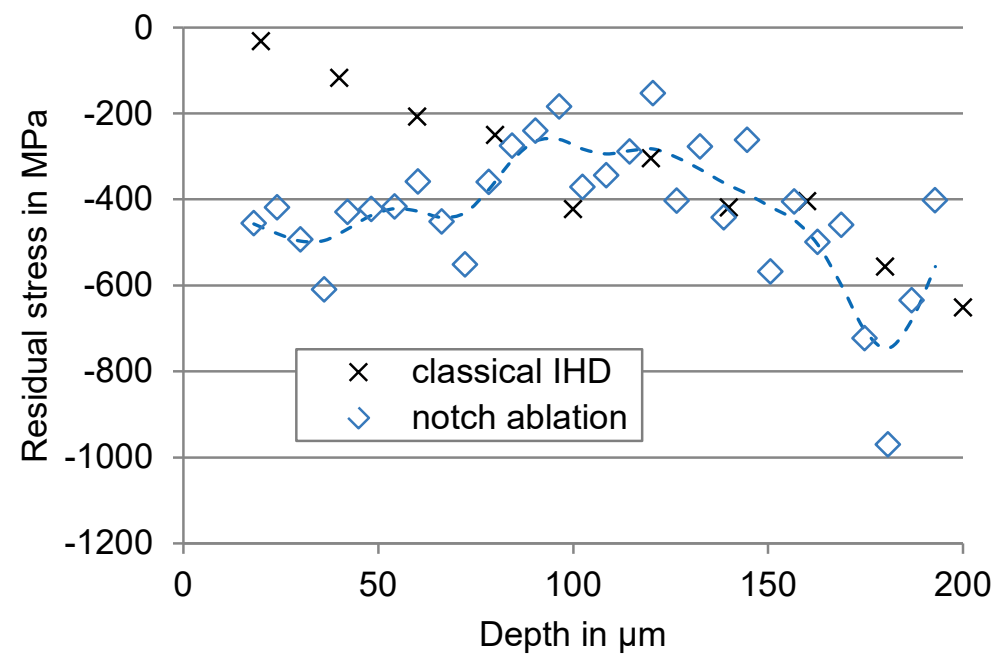

Fig. 5: Residual stress determination with laser notch ablation

Compared to the IHD, the deviation seems higher, which might be related to geometrical inaccuracies and slight depth variations. But solely because of the finer depth steps, small errors lead to bigger notable spreading of results. Noteworthy is also the difference of values up to the depth of $100 \mu \mathrm{m}$. While the IHD shows a linearly rising stress, the notch ablation method indicates surface stresses of roughly $500 \mathrm{MPa}$. A comparative XRD measurement revealed a surface stress of $-596 \pm$ $44 \mathrm{MPa}$. This might indicate a better sensitivity to surface near stresses for the laser notch method. 


\section{Conclusions and outlook}

First results proof the applicability of laser ablation to relax residual stress, where the resulting surface displacements are measured by digital holography and evaluated according to the integral method. The laser ablation seems to imply only neglectable amounts of heat, while the resulting hole geometry can be approximated by simple geometrical forms and, therefore, calibration values can be obtained numerically. A spot focused laser beam leads to some variations in the holes geometry, but overall axial symmetrical removals can be evaluated by using the approach of [5], [6], even if not ideal cylindrical holes and inhomogeneous specimen are present. Hereby, the results of residual stress profiles show the same qualitative behaviour as IHD determinations, but tend to a higher deviation. The main reason is related to uncertainties of the ablated hole structure.

By using SLM to ablate material in notch-form, the structure of removal is significantly more reliable regarding its dimensions. The extensive displacement measurements can be evaluated with a special integral method based scheme and data dependent weighting functions to reduce the corresponding deviation considerably. Hence, especially surface near stresses could be determined more accurate compared to classical IHD determinations. Now continuing analyses should be performed in order to validate the results further.

\section{Acknowledgement}

This work was supported by the German Research Foundation (DFG) under Grant Nos. GA746/10, OS111/37 and SCHM746/120.

\section{References}

[1] P. Weidmann, U. Weber, S. Schmauder, and V. Martinez Garcia, "Investigation of Influence Factors on Residual Stress Determination within Coated Surfaces in Consideration of the Differential and Integral Method," Advanced Materials Research, vol. 996, pp. 307-312, 2014. http://dx.doi.org/10.4028/www.scientific.net/amr.996.307

[2] M. Wenzelburger, D. Lopez, and R. Gadow, "Methods and application of residual stress analysis on thermally sprayed coatings and layer composites," Surface and Coatings Technology, vol. 201, no. 5, pp. 2006. http://dx.doi.org/10.1016/j.surfcoat.2006.04.040

[3] T. Kreis, Handbook of Holographic Interferometry. WILEY-VCH Verlag, 2004. http://dx.doi.org/10.1002/3527604154

[4] P. Weidmann, U. Weber, S. Schmauder, G. Pedrini, and W. Osten, "Numerical calculation of temperature and surface topology during a laser ablation process for ceramic coatings," Meccanica, vol. 51, no. 2, pp. 279-289, 2016. http://dx.doi.org/10.1007/s11012-015-0220-2

[5] G. Schajer and T. Rickert, "Incremental Computation Technique for Residual Stress Calculations Using the Integral Method," Experimental Mechanics, vol. 51, pp. 1217-1222, 2011. http://dx.doi.org/10.1007/s11340-010-9408-5

[6] G. S. Schajer and M. Steinzig, "Full-field calculation of hole drilling residual stresses from electronic speckle pattern interferometry data," Experimental Mechanics, vol. 45, no. 6, pp. 526-532, 2005. http://dx.doi.org/10.1007/BF02427906

[7] G. Pedrini, V. Martinez-Garcia, P. Weidmann, M. Wenzelburger, A. Killinger, U. Weber, S. Schmauder, R. Gadow, and W. Osten, "Residual Stress Analysis of Ceramic Coating by Laser Ablation and Digital Holography," Experimental Mechanics, Dec. 2015.

[8] ASTM837-13, Standard Test Method for Determining Residual Stresses by the Hole-Drilling Strain-Gage Method, no. E 837-13. ASTM, 2013.

[9] G. S. Schajer, "Measurement of Non-uniform Residual Stresses Using the Hole-Drilling Method. Part II - Practical Application of the Integral Method," Transaction of the ASME, vol. 110, pp. 344-349, 1988. http://dx.doi.org/10.1115/1.3226060 